\title{
Machine Learning Era In Heart Disease Prediction- An Intense Learning Analysis With Big Data
}

\author{
Dr. Smrity Prasad ${ }^{1}$ \\ ${ }^{1}$ Department of Computerscience, St. Francis de Sales College, Bengaluru
}

Article History: Received: 11 January 2021; Accepted: 27 February 2021; Published online: 5 April 2021

\begin{abstract}
Machine learning and adaptation is a collection of machine learning methods consisting of several stacked layers and using data to explore hierarchical abstractions. As computer power has increased and large data has emerged, deep learning is an appropriate structure for cardiological tasks. The need to optimize medical treatment varies from diagnostic to therapeutic in the absence of a medical Centre. Machine learning systems are previous attempts to imitate medical practitioners in their protocol for solving medical tasks or for producing observations. These systems are known not to be useful as they require extensive design features and domain expertise in order to achieve the new cardio data highly accurate and difficult to map. Overall, with any technical progress, cardiometry and medicine are autonomous and become closer to an automated, detailed learning area. But no complete conceptual basis for in-depth education can be found. A thorough analysis of its internal functional qualities and constraints is required to enable the field to adopt its position on the disease of the heart.In this study, a large number of very complex machine learning concepts integrated into the cardio domain with big data have been studied over a very short time period of time.
\end{abstract}

Keywords: Machine learning,Implementing, Cardiac, Intense Learning, Big data.

\section{Introduction}

Every year, heart-related diseases claim the lives of about a million people, making this the primary cause. In the United States, heart disease is responsible for one out of every three deaths (US). In 2016, about 9,20,000 people had heart attacks, with about half of them occurring without warning. The only symptom of heart failure is sudden death. A heart attack occurs when the heart's internal organs or muscles begin to expire, even if the individual survives for a short time. This can cause serious complications in the heart's various sections, resulting in health concerns such as an increased risk of cardiac arrest. Coronary heart disease is also known as coronary artery disease (CAD), and is the most common form of heart disease worldwide. Fat storage in the blood veins and arteries causes this ailment. It also prevents blood from flowing into the heart's veins and arteries, resulting in an insufficient supply of oxygen and blood to the heart's internal organs. Angina pectoris, also known as Angina, is a medical term for heart pain caused by a lack of blood flow to the heart. It's a warning sign that you're being checked for heart issues [1].

This form of discomfort can last a few seconds or minutes. Congestive heart failure is a condition in which the heart fails to pump enough blood to the rest of the body's organs. Cardiomyopathy refers to the wearing down of the heart muscles, as well as a change in the form of the muscle, as a result of an inadequate heart pump. Viral infections, alcohol consumption, and hypertension are all common causes of cardiomyopathy [2].The diagnosis and treatment of heart disease is extremely difficult, particularly in developing countries, due to a lack of diagnostic instruments, physicians, and other resources that affect proper cardiac patient prediction and treatment. In response to this issue, computer technology and machine learning techniques have recently been used to build software to assist doctors in making preliminary decisions about heart disease. The death rate can be reduced by early detection of the disease and predicting the likelihood of a person being at risk of heart disease [3].

Medical data mining methods are used to derive useful patterns and information from medical data. Redundancy, multi-attribution, incompleteness, and a close association with time characterize medical data. The health sector faces a major challenge in efficiently using large amounts of data. The methodology and technology for converting these data mounds into valuable decision-making information is provided by data mining. This heart disease prediction system would help cardiologists make faster decisions, allowing more patients to receive care in a shorter amount of time, potentially saving millions of lives [4]. 


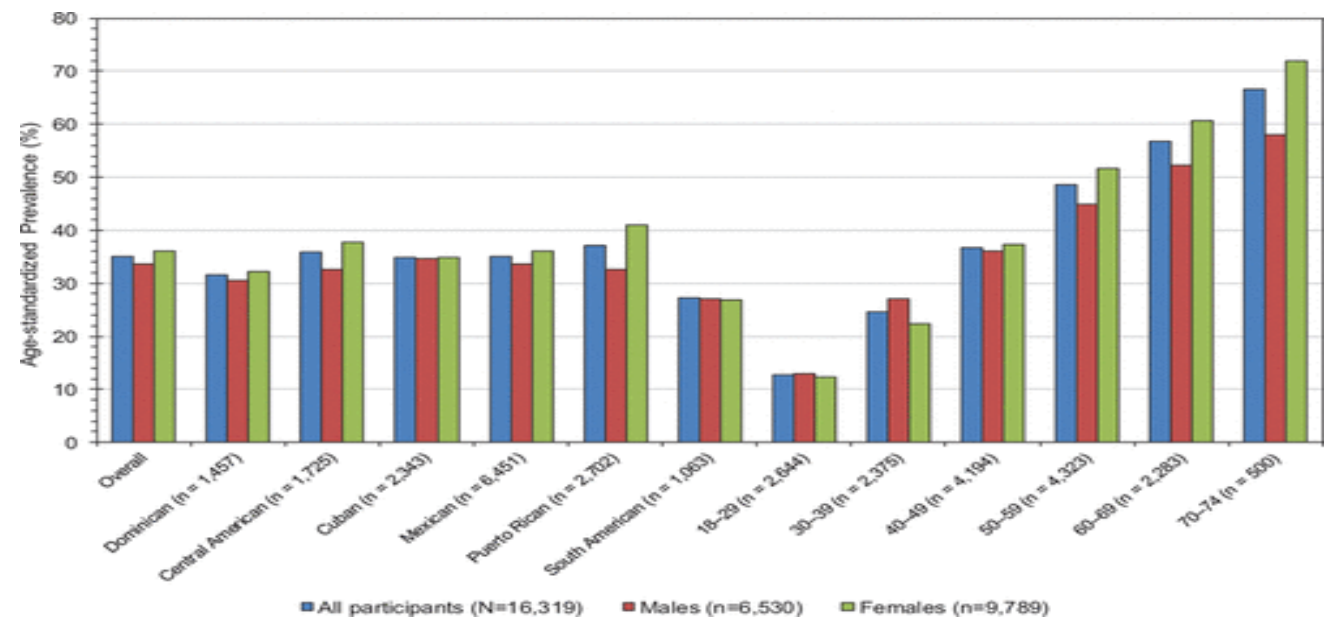

Figure 1. Heart disease meta statistics

Figure 1 depicts the heart disease meta statistics for male and female. Since we have a vast number of medical databases, machine learning will assist us in identifying patterns and extracting useful information from them. Machine learning is mostly used in the medical field for disease prediction, despite its many applications. Machine learning has been used to diagnose heart disease by several researchers because it reduces diagnosis time and increases accuracy and effectiveness. Ample and accurate data is needed for robust machine learning models [5].

The aim of this review is to explain the current state of modern cardiovascular learning and its potential to improve the way we develop and understand knowledge with big data. Section 2 discusses roadmap study of cardiology-related domains, Section 3 discusses big data in cardiovascular imaging, and Section 4 discusses the conclusion.

\section{INTENSE LEARNING OF CARDIOLOGY DOMAIN 2.1 Machine Learning and Clinical Applications}

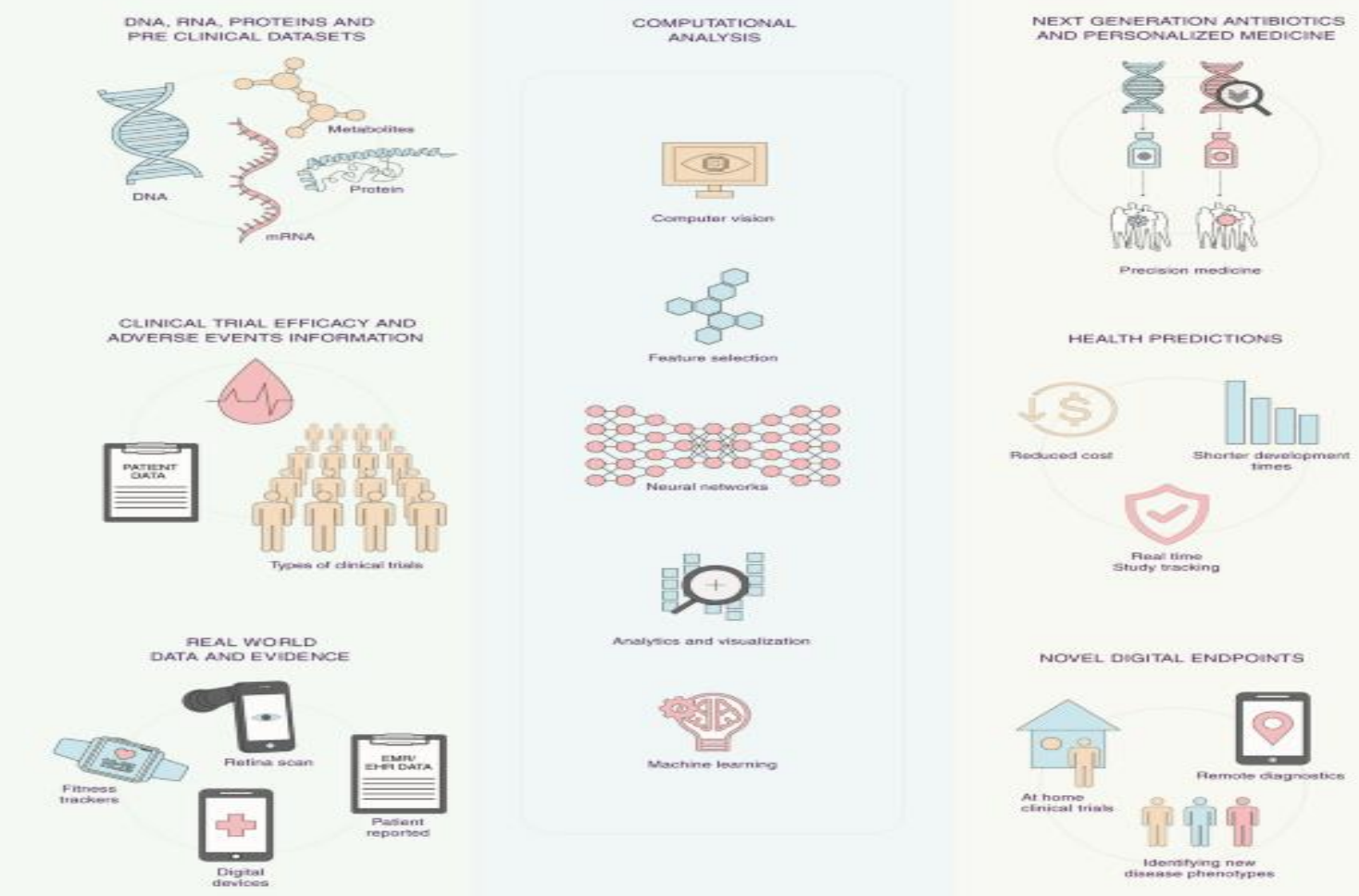

Figure 2. Machine Learning in Clinical Development 
Machine learning approaches from evolutionary psychology is one of the first applications of machine learning in cardiology, in the field of cardiovascular drug therapy. Machine learning, Big Data, and precision medicine have all had a significant impact on the development of new drugs, and are now assisting in the identification of effective treatments while reducing the risk of side effects in a specific individual. Machine learning has been identified as a pathological occurrence in Covid-19 patients in many recent retrospective studies, including cardiac arrhythmias, cardiomyopathy, and cardiac arrest. Higher rates of cardiac arrhythmias, acute coronary psychiatric disorders, and heart failure-related incidents have been observed during seasonal flu outbreaks, suggesting that acute respiratory infections can stimulate coagulation pathways. Another new use of machine learning in cardiovascular immunotherapies and disease control is the treatment of heart failure. Figure 2 depicts the clinical development of machine learning. Machine learning has aided in the development of a newer technique for cardiovascular risk stratification and phenotyping of heart failure, as well as new hypertension and cardiovascular drug therapies and improved medicinal drug therapy [6].

When confounders were taken into account, the primary research looked at the association between preexisting cardiovascular disease and drug treatment and the resulting end of in-hospital death, taking into account demographics and co-existing conditions. Pharmacogenomics and predictive medicine have also had a substantial impact on anticoagulant dosing in different therapeutic classes, as shown by randomised clinical trials.Another use of machine learning in cardiovascular rehabilitation and disease control is the treatment of heart failure. Machine learning has aided in the development of a new approach to cardiovascular risk type division and phenotyping, as well as improved hypertension management, cardiovascular prescription drugs, and medicinal drug therapy. Machine learning was also used to investigate the relationship between cardiac left ventricular systolic reserve function and muscle rigidity in patients with retained ejection fraction in heart failure[7].

\subsection{Implementing Machine Learning in Cardiovascular Applications}

Heart failure with a reduced or retained ejection fraction, multivessel coronary disease, extreme arrhythmias, heart failure, pregnancy cardiovascular disease, or congenital cardiac disease are all common conditions treated by cardiologists. Despite advances in both of these areas, major clinical issues persist. Integrating data from various modes, enabling predictions and refining for special patients with heterogeneous mutations, has some challenges. Machine learning has traditionally been expensive and carried out by scientists using neural networks or high-performance processors. The use of gaming-optimized graphical processing units made profound learning on desktop computers possible due to the demanding nature of machine-learning systems. While professional computing units are still too expensive, machine learning can be done using cloud platforms such as Amazon AWS and Google Cloud. Machine learningsoftware is almost exclusively open source, which means that it is widely available for scientific research with few restrictions [8].

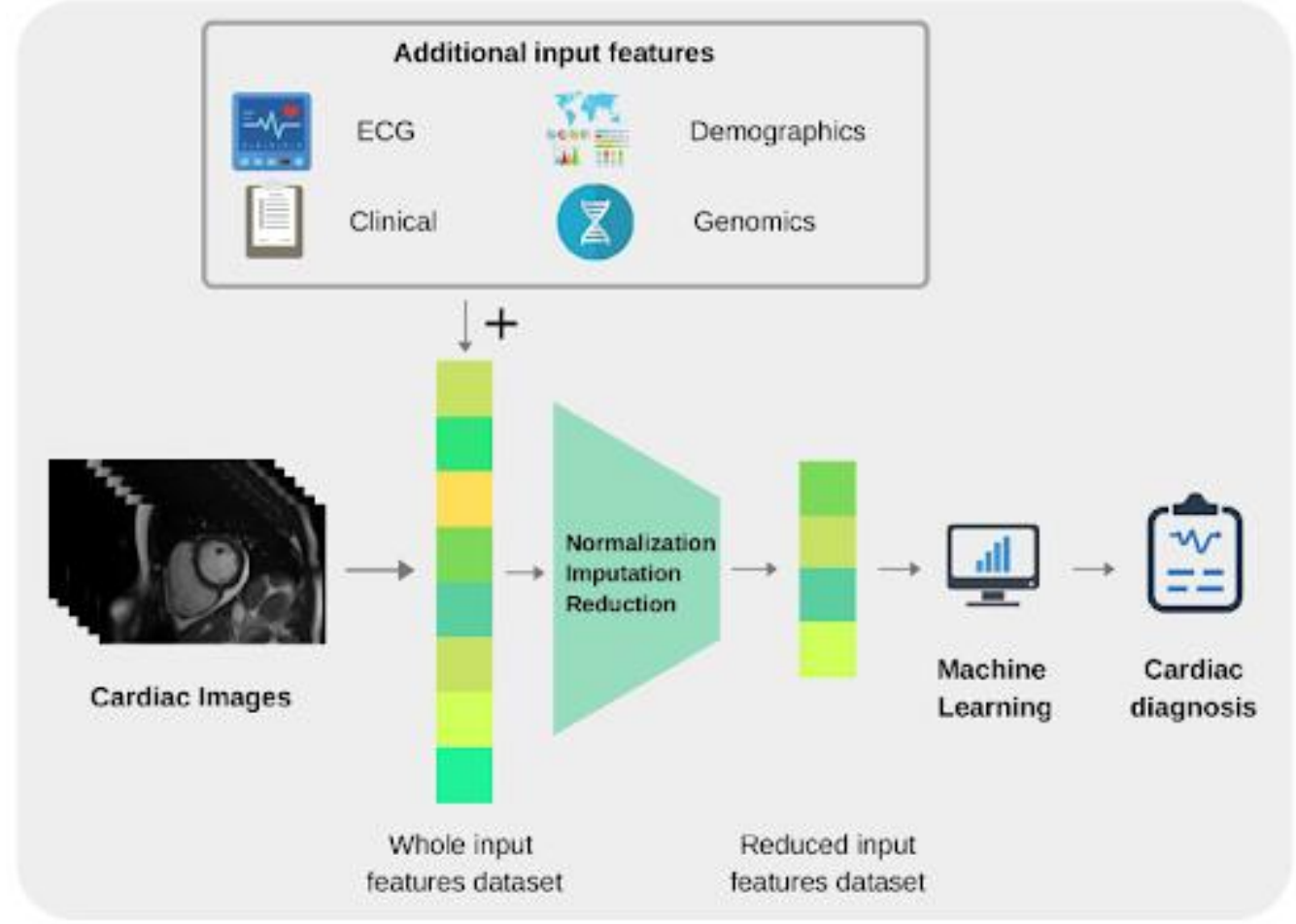

Figure 3. Machine learning in cardiac diagnosis 
Artificial intelligence techniques will become increasingly critical for enhancing cardiologists' workflow and productivity in all aspects of their daily practice. Furthermore, predictive machine learning algorithms may aid in earlier disease detection, improving prognosis. Machine learning is widely used in imaging, but it also has other uses because it allows vast amounts of data to be analyzed rapidly and accurately. This ability can be used to build predictive models for both classification and regression. Over the years, experiments in other fields of medicine have shown AI's promise [9].

\subsection{Cardiac Computed Tomography}

Non-invasive computed tomography imaging, which allows for excellent, high spatial resolution visualization of the coronary arteries, is becoming increasingly popular in the assessment and surveillance of coronary artery disease. In cardiac computed tomography examinations, hundreds of slices are used, and the number of cardiac computed tomography studies has been steadily increasing. As a result of the increased workload for healthcare professionals and a scarcity of skilled cardiac imaging, the facility's cardiac computed tomography has been underutilized. Machine learning can provide a way to resolve these issues and develop automated cardiac computed tomography research with stable and accurate results. Furthermore, machine learning algorithms may allow for a wider range of secondary diagnoses. In cardiac computed tomography of coronary artery disorders and atherosclerosis, machine learning picture treatment approaches are increasingly used in diagnostics and risk assessment [10].

\section{BIG DATA IN CARDIOLOGY}

Patient autonomy in cardiology will be a critical area of progress, particularly for outpatient heart failure care and the reduction of costly readmissions, but also for heart failure and future treatment aspects. Several hurdles remain in the way of improving Big Data's status, including incentives to share data, privacy protection, and data collection confidentiality restrictions. Data analysis should be performed by a specific analyst whose profile is still being developed, but with a pressing need for analytical training, since the rate at which data is generated is rapidly increasing. The clinical practice that is able to design an expressive, intelligent, and goal-oriented interconnections will reap enormous benefits. As part of treatment, cardiovascular medicine offers a variety of physiological, therapeutic, and functional data. These figures are often held in various operational databases that are not easily accessible for cardiovascular research due to technological expertise in digital analysis and personal healings. Machine learning technology's use in cardiovascular medicine, however, is not recent. ECG analysis and scanning are widely being used by specialists as part of in-depth education programs and early approaches to testing and interpreting cardiovascular outcomes [11].

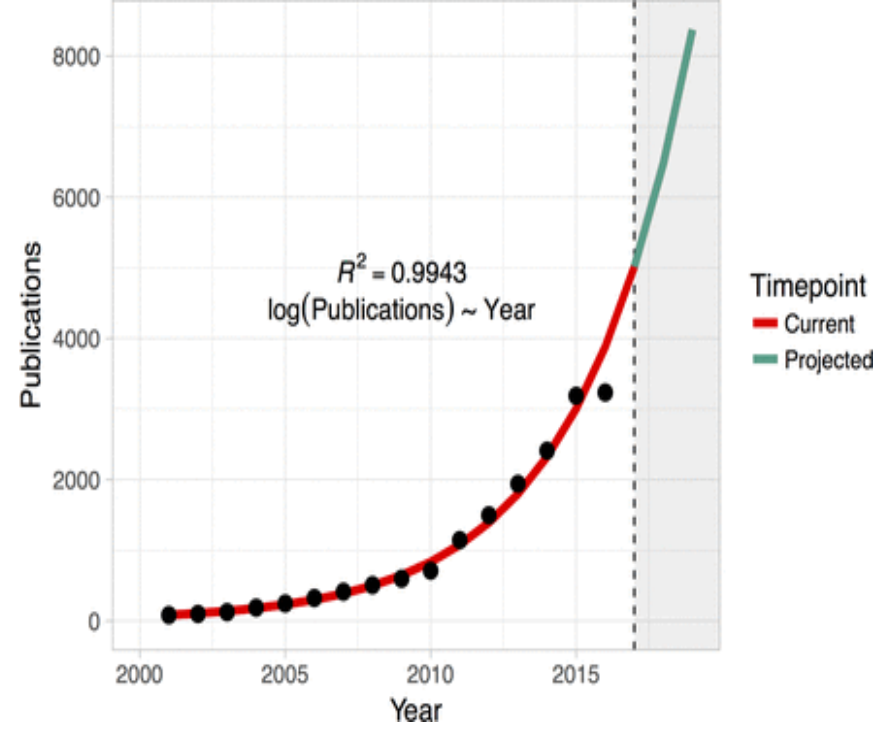

Figure 4. Publications in big data in cardiology

Big data in cardiovascular epidemiology aids research into topographies and various demographic groups, whose density can be modelled by selecting subpopulations in specific areas based on the prevalence analyses' goals. The development of local health programs and the optimum management of ever-dwindling resources are aided by research into the health of the population in small communities. When electronic records for areas or identified populations are available, the data may be linked to hospitalization, allowing for more accurate incident verification and active surveillance. In resource-intensive fields including cardiovascular medicine, physician governance will have to become experienced in assessing such results and recognizing their effects on 
patients. Currently, big data is being used by a number of healthcare organizations, including insurance providers and centers for medicare and medicaidprograms[12].

Big data assists us in developing models that can be used to predict disease occurrence. This knowledge can be used to take preventative measures against the disease. Data assists in disease prevention by offering useful insights. Medical personnel may use machine learning to access medical data and make faster decisions, allowing them to spend more time with patients and reducing cognitive errors. The creation of large data analysis tools for cardiovascular practice and research will continue to grow. Quantitative patient risk modeling and resource utilization, for example, have enormous potential for improving cardiovascular care quality and patient outcomes[13].

Big data analysis approaches are still in their early stages of development and evaluation of cardiovascular care, and there is no evidence that they improve treatment efficiency or patient satisfaction. Large-data methodologies may be more efficient and convenient in cardiovascular applications because they accommodate low-baseline data quality. But, because they result in better data quality, large-data methodologies may be more productive and convenient. In terms of cardiovascular safety and patient outcomes, it's also important to create a 'evidencebaseline' for large-data applications. Furthermore, advances in sensor technology made it possible to reliably translate more aspects of reality into data in small packages while using less energy and spending less money [14].

When taken as a whole, using data today is much less costly than it has ever been. As resource constraints have eroded, methods that based on limited data availability have been reshaped. It is no longer necessary to work with only random experiments to the extent that data can be collected and analyzed accurately and on a large scale.As a result, big data does not necessitate the analysis and processing of millions or even billions of individual measurements. Instead, big database research aims to consider not only a small subset of statistical evidence, but a large portion, if not all, of them [15].

\section{CONCLUSION}

Despite significant advances in diagnosis and treatment, cardiovascular disease remains the leading cause of morbidity and mortality worldwide, accounting for nearly one-third of all deaths. Early and accurate diagnosis is the secret to better cardiovascular functional outcomes. To improve the diagnostic utility of cardiac imaging, more sophisticated image processing techniques are required, allowing for more detailed measurements of imaging phenotypes. In recent years, the exponential growth of machine learning techniques in medical imaging has been fueled by the development of large amounts of data and the availability of large amounts of computing resources. As a result, numerous machine learning perspectives integrated into the cardio domain are explored in this analysis.

\section{References}

1. Al'Aref, Subhi \&Anchouche, Khalil \& Singh, Gurpreet \&Slomka, Piotr \&Kolli, Kranthi\& Kumar, Amit \& Pandey, Mohit \&Maliakal, Gabriel \& van Rosendael, Alexander \&Beecy, Ashley \& Berman, Daniel \& Leipsic, Jonathan \& Nieman, Koen \&Andreini, Daniele \&Pontone, Gianluca \&Schoepf, Uwe \& Shaw, Leslee\& Chang, Hyuk-Jae \& Narula, Jagat \& Min, James. (2018). Clinical applications of machine learning in cardiovascular disease and its relevance to cardiac imaging. European heart journal. 40. 10.1093/eurheartj/ehy404.

2. Amani, Reza \& Sharifi, Nasrin. (2012). Cardiovascular Disease Risk Factors. 10.5772/34374.

3. Bernard, Olivier \&Lalande, Alain \&Zotti, Clement \&Cervenansky, Frederick \& Yang, Xin \& Heng, Pheng-Ann \& Cetin, Irem\&Lekadir, Karim \& Camara, Oscar \& González Ballester, Miguel Ángel\&Sanroma, Gerard \&Napel, Sandy \& Petersen, Steffen \&Tziritas, Georgios \&Ilias, Grinias\&Khened, Mahendra\&Kollerathu, Varghese \&Krishnamurthi, Ganapathy \&Rohe, Marc-Michel \&Jodoin, Pierre-Marc. (2018). Deep Learning Techniques for Automatic MRI Cardiac Multi-Structures Segmentation and Diagnosis: Is the Problem Solved?. IEEE Transactions on Medical Imaging. PP. 1-1. 10.1109/TMI.2018.2837502.

4. Benjamins, Jan Walter \& Hendriks, Tom \&Knuuti, Juhani \& Juárez-Orozco, Luis \& van der Harst, Pim. (2019). A primer in artificial intelligence in cardiovascular medicine. Netherlands Heart Journal. 27. 10.1007/s12471-019-1286-6.

5. Bhat, Mudasir\& Ahmad, S. \&Ganai, Nazir \& Shah, Riaz \& Zaman, Majid \& Khan, Sameer \& Shah, Aftab. (2021). Prediction of Cardiovascular Disease Through Cutting-Edge Deep Learning Technologies: An Empirical Study Based on TENSORFLOW, PYTORCH and KERAS. 10.1007/978981-15-5113-0_18.

6. Bizopoulos, Paschalis \&Koutsouris, Dimitris. (2018). Deep Learning in Cardiology. IEEE Reviews in Biomedical Engineering. PP. 1-1. 10.1109/ RBME. 2018.2885714. 
7. Bommadevara, H.S.A. \& Sowmya, Y. \& Gera, Pradeepini. (2019). Heart disease prediction using machine learning algorithms. International Journal of Innovative Technology and Exploring Engineering. 8. 270-272.

8. Cao, Yankun\& Liu, Zhi\& Zhang, Pengfei\& Zheng, Yushuo\& Song, Yongsheng\& Cui, Lizhen. (2019). Deep Learning Methods for Cardiovascular Image. Journal of Artificial Intelligence and Systems. 1. 96109. 10.33969/AIS.2019.11006.

9. Darmawahyuni, Annisa\&Nurmaini, Siti \& Firdaus, Firdaus. (2019). Coronary Heart Disease Interpretation Based on Deep Neural Network. Computer Engineering and Applications Journal. 8. 112. 10.18495/comengapp.v8i1.288.

10. Debabrata Mukherjee, "Cardiovascular and Hematological Medicine in 2019 - Advances and Insights ", Cardiovascular \& Hematological Agents in Medicinal Chemistry (2019) 17: 2. https://doi.org/10.2174/187152571701190722113042

11. Doppala, Bhanu Prakash \& Bhattacharyya, Debnath \&Chakkravarthy, Midhun. (2020). Stratification of Cardiovascular Diseases Using Deep Learning. Revue d intelligence artificielle. 34. 377-385. 10.18280/ria.340401.

12. Eid, Marwen\& Spearman, James \& van Assen, Marly \& De Santis, Domenico \&Sahbaee, Pooyan\& Landreth, Scott \& Jacobs, Brian \& De Cecco, Carlo N.. (2019). Machine Learning and Artificial Intelligence in Cardiovascular Imaging. 10.1007/978-1- 60327- 237- 7_68.

13. Gogi, Giovanah\&Gegov, Alexander. (2020). Application of Deep Learning for the Diagnosis of Cardiovascular Diseases. 10.1007/978-3-030 -29516-5_59.

14. Gopika, P. \&Vishvanathan, Sowmya \& Gopalakrishnan, E. A \&Kp, Soman. (2020). Transferable approach for cardiac disease classification using deep learning. 10.1016/B978-0-12-819061-6.00012-4.

15. Hajar, Rachel. (2016). Framingham Contribution to Cardiovascular Disease. Heart Views. 17. 78. $10.4103 / 1995-705 X .185130$. 